\title{
INDEX TO VOLUME 72
}

\section{RESEARCH ANNOUNCEMENTS}

Allen, H. P. Jordan algebras and Lie algebras of type $D_{4}, 65$.

Anderson, D. R. A property of the $L_{2}$-norm of a convolution, 693.

Anderson, D. W., Brown, E. H. Jr. and Peterson, F. P. Spin cobordism, 256.

Anderson, R. D. Hilbert space is homeomorphic to the countable infinite product of lines, 515.

Arraut, J. L. Note on structural stability, 542.

Assmus, E. F. Jr. and Mattson, H. F. Jr. Disjoint Steiner systems associated with the Mathieu groups, 843.

Atiyah, M. F. and Bott, R. A Lefshetz fixed point formula for elliptic differential operators, 245.

Bailey, Paul, Shampine, Lawrence F. and Waltman, Paul. Existence and uniqueness of solutions to the second order boundary value problem, 96.

Balogh, Charles B. Uniform asymptotic expansions of the modified Bessel function of the third kind of large imaginary order, 40.

Baumert, L. D. Hadamard matrices of orders 116 and 232, 237.

Beals, Richard. On eigenvalue distributions for elliptic operators without smooth coefficients, 701.

Berger, M. S. and Fife, P. C. On Von Karman's equations and the buckling of a thin elastic plate, 1006.

- and Meyers, N. G. On a system of nonlinear partial differential equations arising in mathematical economics, 954.

Bishop, R. L. and Goldberg, S. I. A characterization of the euclidean sphere, 122.

Boardman, J. M. and Steer, B. Axioms for Hopf invariants, 992.

Boas, R. P. Jr. Fourier series with positive coefficients, 863.

Bogdanowicz, W. M. Multilinear Lebesgue-Bochner-Stieltjes integral, 232.

- Integral representation of multilinear continuous operators from the space of Lebesgue-Bochner summable functions into any Banach space, 317.

Bott, R. See Atiyah, M. F.

Browder, F. E. Nonlinear elliptic functional equations in nonreflexive Banach spaces, 89. and Petryshyn, W. V. The solution by iteration of linear functional equations in Banach spaces, 366.

- The solution by iteration of nonlinear functional equations in Banach spaces, 571 .

Browder, William. Embedding 1-connected manifolds, 225; 736.

Manifolds with $\pi_{1}=Z, 238$.

and Hirsch, M. W. Surgery on piecewise linear manifolds and applications, 959.

Brown, E. H. Jr. See Anderson, D. W.

Brumer, Armand. Pseudocompact algebras, profinite groups and class formations, 321.

Butzer, P. L. and Nessel, R. J. Favard classes for n-dimensional singular integrals, 493.

Carroll, Robert. On the nature of weak solutions and some abstract Cauchy problems, 1068.

Clark, Colin. An asymptotic formula for the eigenvalues of the Laplacian operator in an unbounded domain, 709.

Cohen, J. M. Some results on the stable homotopy groups of spheres, 732.

Conway, J. B. The strict topology and compactness in the space of measures, 75. 
Subspaces of $C(S)_{\beta}$, the space $\left(l^{\infty}, \beta\right)$, and $\left(H^{\infty}, \beta\right), 79$.

Cooke, K. L. Functional differential equations close to differential equations, 285.

Dedecker, Paul and Lue, A. S.-T. A non-Abelian two-dimensional cohomology for associative algebras, 1044.

Denzel, G. E. The hitting characteristics of a strong Markov process, with applications to continuous Martingales in $R^{n}, 1026$.

Fabes, E. B. and Rivière, N. M. Parabolic partial differential equations with uniformly continuous coefficients, 116.

Federer, Herbert. Two theorems in geometric measure theory, 719.

Feferman, S. Predicative provability in set theory, 486.

- and Kreisel, G. Persistent and invariant formulas relative to theories of higher order, 480.

Fife, P. C. See Merger, M. S.

Fisher, L. D. Jr. The convex hull of a sample, 555.

Fossum, R. M. The Noetherian different of projective orders, 898.

Fox, R. H. Rolling, 162.

Franck, W. E. and Hanson, D. L. Some results giving rates of convergence in the law of large numbers for weighted sums of independent random variables, 266.

Frederickson, P. O. See Moeller, J. W.

Freeman, R. S. On the spectrum and resolvent of homogeneous elliptic differential operators with constant coefficients, 538.

Friedman, Avner. Solvability of the first Cousin problem and vanishing of higher cohomology groups for domains which are not domains of holomorphy. II, 505.

Freilich, Gerald. Centers of curvature, 297.

Fuller, F. B. The treatment of periodic orbits by the methods of fixed point theory, 838 .

Garnett, John. Disconnected Gleason parts, 490.

Gilbert, R. P. and Howard, H. C. A generalization of a theorem of Nehari, 37.

Glaser, L. C. Intersections of combinatorial balls and of Euclidean spaces, 68.

Goffman, Casper. Linearly continuous functions of finite area, 1055.

Goldberg, S. I. See Bishop, R. L.

Graham, Phyllis. Cohomology of dihedral groups of order $2 p, 324$.

Granirer, E. E. Extremely amenable semigroups, 1028.

Green, L. W. The Pythagorean group and ergodic flows, 44.

Greenberg, Marvin. Rational points in Henselian discrete valuation rings, 713.

Gross, Fred. On the equation $f^{n}+g^{n}=1,86 ; 576$.

Grosser, Siegfried and Moskowitz, Martin. On central topological groups, 826.

Representation theory of central topological groups, 831.

Gustafson, Karl. A perturbation lemma, 334 .

Hager, A. W. On the maximal ring of quotients of $C(X), 850$.

Halkin, Hubert. An abstract framework for the theory of process optimization, 677.

Hanson, D. L. See Franck, W. E.

Heins, A. E. Errata to "Axially-symmetric boundary-value problems," 342.

Hinrichsen, Diederich. Adapted integral representations by measures of Choquet boundaries, 888.

Hirsch, M. W. and Zeeman, E. C. Engulfing, 113.

Hirsch, M. W. See Browder, William.

Holm, Per. Microbundles and bundles, 545.

Microbundles and Thom classes, 549.

Howard, H. C. See Gilbert, R. P.

Hsiang, Wu-chung. Locally flat nonembeddability of certain parellelizable manifolds, 227. 
and Hsiang, Wu-yi. Some results on differentiable actions, 134.

Hsiang, Wu-yi. See Hsiang Wu-chung.

Hudson, J. F. P. Concordance and isotopy of PL embeddings, 534.

Piecewise linear embeddings and isotopies, 536.

Husch, L. S. On regular neighborhoods of spheres, 879.

Jenkins, Howard and Serrin, James. The Dirichlet problem for the minimal surface equation, with infinite data, 102.

Johnson, A. A. Integral representations of Hermitian forms over local fields, 118.

Juberg, R. K. On the oscillations and Lebesgue classes of a function and its potentials, 157.

Kadison, R. V. and Ringrose, J. R. Automorphisms of operator algebras, 1059.

Kahn, P. J. Self-equivalences of (n-1)-connected $2 n$-manifolds, 562.

Kamber, F. and Tondeur, P. On flat bundles, 846.

Kaufman, Robert. Examples in Helson sets, 139.

- and Rickert, N. W. An inequality concerning measures, 672.

Keisler, H. J. First order properties of pairs of cardinals, 141.

Kelleher, James. Rings of meromorphic functions, 54.

Keller, H. B. Existence theory for two point boundary value problems, 728.

Keynes, H. B. Topological dynamics in coset transformation groups, 1033.

Kirby, R. C. Smoothing locally flat embeddings, 147.

Kraines, David. Higher products, 128.

Kreisel, G. See Feferman, S.

Krengel, Ulrich. Remark on the modulus of compact operators, 132.

Lander, L. J. and Parkin, T. R. Counterexample to Euler's conjecture on sums of like powers, 1099.

Lashof, R. and Rothemberg, M. On the Hauptvermutung, triangulation of manifolds, and $h$-cobordism, 1040.

Leptin, Horst. On certain invariant of a locally compact group, 870.

Lindenstrauss, Joram. On nonseparable reflexive Banach spaces, 967.

Loeb, Peter. See Walsh, Bertram.

Lue, A. S. T. See Dedecker, Paul.

MacAlpine, E. A. M. Duality in a twisted homology theory, 1051.

Marden, A. and Rodin, B. A complete extremal distance problem on open Riemann surfaces, 326.

Mattson, H. F. Jr. See Assmus, E. F. Jr.

Meyers, N. G. See Berger, M. S.

Miller, R. K. Asymptotic behavior of solutions of nonlinear Volterra equations, 153.

Moeller, J. W. and Frederickson, P. O. A density theorem for lacunary Fourier series, 82.

Moskowitz, Martin. See Grosser, Siegfried.

Nakai, Mitsuru. Royden's map between Riemann surfaces, 1003.

- and Sario, Leo. Normal operators, linear liftings, and the Wiener compactification, 947.

Nessell, R. J. See Butzer, P. L.

Newman, D. J. and Shapiro, H. S. Certain Hilbert spaces of entire functions, 971.

Newman, M. H. A. The engulfing theorem for locally tame sets, 861.

Oklander, E. T. $L_{p q}$ interpolators and the theorem of Marcinkiewicz, 49.

Owens, F. W. Extensions of commuting isotone functions, 965.

Parkin, T. R. See Lander, L. J.

Parry, William. Generators and strong generators in ergodic theory, 294. 
Parthasarathy, K. R., Ranga Rao, R. and Varadarajan, V. S. Representation of complex semisimple Lie groups and Lie algebras, 522.

Pendleton, R. L. A characterization of Q-domains, 499.

Peleg, Bezalel. The independence of game theory of utility theory, 995.

Peterson, F. P. See Anderson, D. W.

Petrie, T. The $K$ theory of the projective unitary groups, 875 .

Petryshyn, W. V. On a fixed point theorem for nonlinear P-compact operators in Banach space, 329.

See Browder, F. E.

Pinchuk, Bernard. Extremal problems in the class of close-to-convex functions, 1014.

Pincus, J. D. The spectral theory of self-adjoint Wiener-Hopf operators, 882.

Ponomarenko, Paul. Errata to "The Galois theory of infinite purely inseparable extensions," 342.

Protter, M. H. and Weinberger, H. F. On the spectrum of general second order operators, 251.

Quintas, L. V. and Supnick, Fred. Extrema concerning polygons in space-time, 71.

Ralley, Thomas. Decomposition of products of modular representations, 1012.

Ranga Rao, R. See Parthasarathy, K. R.

Reneke, J. A. Linear functions on the space of quasicontinuous functions, 1023.

Rider, Daniel. A relation between a theorem of Bohr and Sidon sets, 558.

Central idempotents in group algebra, 1000.

Richards, Ian. On Malliavin's counterexample to spectral synthesis, 698.

Rickert, N. W. See Kaufman, R. P.

Ringrose, J. R. See Kadison, R. V.

Riordan, John. The enumeration of labeled trees by degrees, 110.

Riviere, N. M. See Fabes, E. B.

Rodin, Burton. Extremal length and removable boundaries of Riemann surfaces, 274. See Marden, A.

Rothenberg, M. See Lashof, R.

Rourke, C. P. and Sanderson, B. J. Block bundles, 1036.

Rubel, L. A. and Taylor, B. A. A Fourier series method for meromorphic and entire functions, 858.

Rudin, Walter. Ideals with small automorphisms, 339.

Zeros and factorizations of holomorphic functions, 1064.

Sacks, G. E. Metarecursively enumerable sets and admissible ordinals, 59.

Sakai, Shoichiro. On a characterization of type I $C^{*}$-algebras, 508.

Sally, P. J. Jr. Uniformly bounded representations of the universal covering group of $\mathrm{SL}(2, R), 269$.

Sanderson, B. J. See Rourke, C. P.

Sario, Leo. See Nakai, Mitsuru.

Sato, Daihachiro and Straus, E. G. Generalized interpolation by analytic functions, 32.

Savage, Nevin. Ahlfors' conjecture concerning extreme Sario operators, 720.

Schaufele, C. B. A note on link groups, 107.

Schechter, Martin. Interpolation spaces by complex methods, 526.

Schneider, W. J. An entire transcendental function whose inverse takes sets of finite measure into sets of finite measure, 841.

Schönbeck, S. O. Extension of nonlinear contractions, 99.

Schultz, M. H. On the stability of discretizations, 950.

Segal, Jack. Isomorphic complexes. II, 300.

Sell, G. R. Almost periodic and periodic solutions of difference equations, 261. 
Serrın, James. See Jenkins, Howard.

Shamir, Eliahu. Wiener-Hopf type problems for elliptic systems of singular integral equations, 501.

Shampine, L. F. See Bailey, Paul.

Shapiro, H. S. See Newman, D. J.

Shatz, S. S. Grothendieck topologies over complete local rings, 303.

Shih, Weishu. Fiber cobordism and the index of a family of elliptic differential operators, 984.

Sidák, Zbyněk. Classification of Markov chains with a general state space, 149.

Small, L. W. On some questions in Noetherian rings, 853.

Sobczyk, Andrew. Some unsymmetric combinatorial numbers, 512.

Solovay, R. M. New proof of a theorem of Gaifman and Hales, 282.

Stampfli, J. G. Normality and the numerical range of an operator, 1021.

Steer, B. See Boardman, J. M.

Steiner, A. K. The topological complementation problem, 125.

Stenger, William. On Poincare's bounds for higher eigenvalues, 715.

Straus, E. G. See Sato, Daihachiro.

Sumners, D. W. L. Higher dimensional slice knots, 894.

Sundaresan, Kondagunta. Smooth Banach spaces, 520.

Supnick, Fred. See Quintas, L. V.

Tait, W. W. A nonconstructive proof of Gentzen's Hauptsatz for second order predicate $\operatorname{logic}, 980$.

Taylor, B. A. See Rubel, L. A.

Thomas, C. B. The oriented homotopy type of 3-manifolds, 291.

Tindell, Ralph. A counterexample on relative regular neighborhoods, 892.

Togo, Shigeaki. Derivations of Lie algebras, 690.

Ton, Bui An. On nonlinear elliptic boundary-value problems, 307.

Tondeur, $\mathrm{Ph}$. see Kamber, $\mathrm{F}$.

Ullman, J. L. A class of weight functions for which Tchebycheff quadrature is possible, 1073.

deValcourt, A. B (Brother). Measures of axial symmetry for ovals, 289.

Varadarajan, V. S. See Parthasarathy, K. R.

Walsh, Bertram and Loeb, P. A. Nuclearity in axiomatic potential theory, 685.

Waltman, Paul. See Bailey, Paul.

Warne, R. J. On certain bisimple inverse semigroups, 679. Extensions of Brandt semigroups, 683.

Wasserman, Arthur. Cobordism of group actions, 866.

Weinberger, H. F. See Protter, M. H.

Whitfield, J. H. M. Differentiable functions with bounded nonempty support on Banach spaces, 145.

Winter, D. J. On automorphisms of algebraic groups, 706.

$\mathrm{Wu}, \mathrm{T}$. S. Left almost periodicity does not imply right almost periodicity, 314.

Yang, C. H. A construction for maximal (+1, -1)-matrix of order 54, 293.

Zeeman, E. C. See Hirsch, M. W.

Zemanian, A. H. The convolution transformation of certain distributions and its inversion, 725 . 


\section{RESEARCH PROBLEMS}

Bellman, Richard. Lagrange expansion for functionals, 220. Singular differential and the Lagrange expansion, 220.

Truncation of infinite system of ordinary differential equations, 470.

Brauer, G. U. Sets of convergence of exponential series, 470.

Charmonman, Srisakdi. Eigenvalues of $2 n \times 2 n$ matrix, 222.

DeMarr, Ralph. Complete lattices and compact Hausdorff spaces, 223.

Ezeilo, J. O. C. Periodic solutions of differential equations, 470.

Flor, Peter. Matrix theory, 30.

Gandhi, J. M. The number of representations of a number as a sum of ten squares, 220.

Gross, Fred. Analytic functions, 223.

On periodic entire functions, 656.

Klee, Victor and Wilansky, Albert, 656.

Mullin, A. A. Word-problems in metamathematics, 656.

Redheffer, R. M. Three problems in elementary analysis, 221.

Spria, Robert. Riemann hypothesis in function fields, 30.

Wilansky, Albert. See Klee, Victor.

\section{BOOK REVIEWS}

Hilton, Peter. Addendum to "Review of Modern algebraic topology by D. G. Bourgin," 736.

Weiss, Edwin. Algebraic number theory. Ronald Jacobowitz, 466.

\section{REPORTS OF MEETINGS AND MISCELLANEOUS ARTICLES}

Bibliography of Norbert Wiener, 135.

Brelot, M. Norbert Wiener and potential theory, 39.

Calderón, A. P. Singular integrals, 427.

Chern, S. S. The geometry of G-structures, 167.

Council and Board of Trustees-1965, 165.

Doob, J. L. Wiener's work in probability theory, 69.

Green, J. W. and Sherman, Seymour. Reports of Meetings of the American Mathematical Society: The Annual Meeting in Chicago, 472; The April Meeting in Chicago, 659.

Harrold, O. J. Reports of Meetings of the American Mathematical Society: The November Meeting in Lexington, 31.

Kac, M. Wiener and integration in function spaces, 52.

Kahane, J. P. Norbert Wiener et l'analyse de Fourier, 42.

Lehmer, D. H. Mechanized mathematics, 739.

Levinson, N. Wiener's life, 1.

Mandelbrojt, S. Les taubériens généraux de Norbert Wiener, 48.

Masani, P. Wiener's contributions to generalized harmonic analysis, prediction theory and filter theory, 73.

Milnor, J. Whitehead torsion, 358.

Peirce, R. S. Reports of Meetings of the American Mathematical Society: The December Meeting in Berkeley, 471; The April Meeting in Hawaii, 824.; The June Meeting in Victoria, 825.

Pitcher, Everett. Reports of Meetings of the American Mathematical Society: The February Meeting in New York, 657; The April Meeting in New York, 658; The Summer Meeting in New Brunswick, 938. 
Root, W. L. Contributions of Norbert Wiener to communication theory, 126.

Rosenblith, Walter and Wiesner, Jerome. From philosophy to mathematics to biology, 33.

Sherman, Seymour. Reports of Meetings of the American Mathematical Society: The November Meeting in Iowa, 224; see J. W. Green.

Wiesner, Jerome. See Rosenblith, Walter.

\section{INVITED ADDRESSES}

Askey, Richard. Norm inequalities for some orthogonal series, 808.

Doob, K. L. Applications to analysis of a topological definition of smallness of a set, 579.

Eells, James Jr. A setting for global analysis, 751

Isbell, J. R. Structure of categories, 619.

Mostert, P. S. The structure of topological semigroups-revisited, 601.

Koecher, Max. On homogeneous algebras, 347.

Lorentz, G. G. Metric entropy and approximation, 903.

Nijenhuis, Albert and Richardson, R. W. Jr. Cohomology and deformations in graded Lie algebras, 1.

Richardson, R. W. Jr. See Nijenhuis, Albert.

\section{STATEMENT OF OWNERSHIP, MANAGEMENT AND CIRCULATION}

Act of October 23, 1962 : Section 4369, Title 39, United States Code

1. Date of Filing: September 23, 1966

2. Title of Publication: Bulletin of the American Mathematical Society

3. Frequency of issue: Six times per year

4. Location of Known Office of Publication: P. O. Box 6248, Providence, Rhode Island 02904

5. Location of the Headquarters or General Business Offices of the Publishers: Same

6. Names and Addresses of Publisher, Editor, and Managing Editor. Publisher: American Mathematical Society, P. O. Box 6248, Providence, Rhode Island 02904. Editor: Felix Browder, Chairman of the Editorial Committee, P. O. Box 6248, Providence, Rhode Island 02904. Managing Editor: None

7. Owner: None

8. Known Bondholders, Mortgagees and Other Security Holders Owning or Holding 1 Percent or More of Total Amount of Bonds, Mortgages or Other Securities: None

9. Paragraphs 7 and 8 include, in cases where the stockholder or security holder appears upon the books of the company as trustee or in any other fiduciary relation, the name of the person or corporation for whom such trustee is acting, also the statements in the two paragraphs show the affiant's full knowledge and belief as to the circumstances and conditions under which stockholders and security holders who do not appear upon the books of the company as trustees, hold stock and securities in a capacity other than that of a bona fide owner. Names and addresses of individuals who are stockholders of a corporation which itself is a stockholder or holder of bonds, mortgages or other securities of the publishing corporation have been included in paragraphs 7 and 8 when the interests of such individuals are equivalent to 1 percent or more of the total amount of the stock or securities of the publishing corporation.

I certify that the statements made by me above are correct and complete.-Gordon L. Walker 